\title{
TIME DELAY TEMPERATURE CONTROL WITH IMC AND CLOSED-LOOP IDENTIFICATION
}

\author{
Naoto ABE* ${ }^{*}$ Katsuyoshi KARAKAWA* and Hiroyuki ICHIHARA ${ }^{\dagger}$ \\ * Department of Mechanical Engineering Informatics, Meiji University Kawasaki 214-8571, JAPAN \\ abe@ messe.meiji.ac.jp TEL: +81-44-934-7358 FAX: +81-44-934-7907 \\ $\dagger$ Department of Control Engineering and Science, Kyusyu Institute of Technology, Iizuka-shi Fukuoka 820-8502, JAPAN \\ ichihara@ces.kyutech.ac.jp TEL: +81-948-29-7500 FAX: +81-948-29-7517
}

Keywords: Closed-loop Identification, Time-delay system, Internal Model Control, Joint Design Method, Frigistor Module

\begin{abstract}
For systems with time delay, several control strategies have been developed, for example, Smith predictor control, IMC (Internal Model Control), LQG and so on. IMC is one of the simple design methods for systems with time delay, however, the above model based controller requires exactly parameter, including the value of time delay. Time delay systems exist in the process control and it is difficult for the process system to make a mathematical model. In order to derive a good closedloop property, closed-loop identification is one of the strongly strategy. This paper deals with experimental results for a cooling temperature control systems with an input time delay by using a joint design method of closed-loop identification and IMC. The results show that the closed-loop has good performance with easily parameter setting compared with ordinary PID control.
\end{abstract}

\section{Introduction}

Time delay has been common phenomenon to feedback control systems and it is difficult to control. Smith predictor control is well known strategy for time delay systems. The scheme is based on the prediction of the system output of after the time delay period. LQG for time delay systems and IMC (Internal Model Control) are also depend on the prediction of the system behavior.

For the prediction of the system behavior, it is strongly required to the precise parameters of the plant. In process control systems such as a chemical process and a temperature control, it is known that to make a mathmatical nominal model is hard working. Therefore, a closed-loop identification method is natural choice for taking care of the closed-loop property.

Recently, some iterative methods of high quality compensator, which is repeatedly designed with closed-loop identifications, has been developed $[3,6,8,9,10]$. In these methods, a compensator was re-designed by the $\mathcal{H}_{2}$ control on the basis of a re-identified model derived from the closed-loop identification. The re-identified model had a closed-loop property and information of the last compensator, and the pre information were used the next design step. We applied these methodology to the vibration control system [4].

In the case of the iterative design method of IMC structure for lumped parameter systems, the windsurfer approach is known [7]. When nominal model and closed-loop property (IMC filter) are decided, IMC controller is immediately designed without complicated manipulations [11]. The windsurfer approach uses the fractional representation approach as the closed-loop identification [12]. IMC has also advantage to use to the process control, that is, the closed-loop bandwidth can be easily set by the only one free parameter.

An iterative design method of LQG for time delay systems has been developed [2]. At that case, the procedure of lumped parameter case e.g. [9] could not be applied directly, because of it needed a spectrum factorization with integral kernel. The problem was avoided by using the predictive virtual system. However, it had not only the identification problem but also how to choose the cost function to apply the control plants.

The application of windsurfer approach for time delay systems has also some problems. When the IMC structure with the fractional closed-loop identification [12] is used for systems with an input time delay, the re-identified model is not in the same class of the input time delay systems and the model can not be used to design IMC controller directly. This problem was solved by using the fixed DARX model, which had the fixed order lumped part and an input time delay. Time delay was decided by sum of the square of predictive error with the cross validation and lumped part is identified by the ordinary least square method with pre-filter [5].

In this paper, experimental results of the proposed iterative design method for the cooling temperature control system by the frigistor module. The process looks like very simple and it looks easy to control, however, the simple open-loop identification of the step response makes insufficient model. The iterative design method has good closed-loop property.

In section 2, we introduce the algorithm of joint design method of the closed-loop identification and IMC structure for systems with an input time delay. In section 3, we show experimental results of the method for a cooling temperature control system with an input time delay. 


\section{Joint Design Method for Time Delay System}

\subsection{IMC structure method [11]}

IMC is one of the simple design method for systems with time delay. The feature of IMC is that the model of plant is included in feedback control loop. The closed-loop property is given in the following;

$$
F(s):=\frac{1}{(\lambda s+1)^{n}} e^{-s h}
$$

when $\lambda$ is set smaller, then the band width becomes larger. $n$ is an integer decided by the relative order of the plant to make a proper IMC controller. $h$ denotes the time delay of the plant.

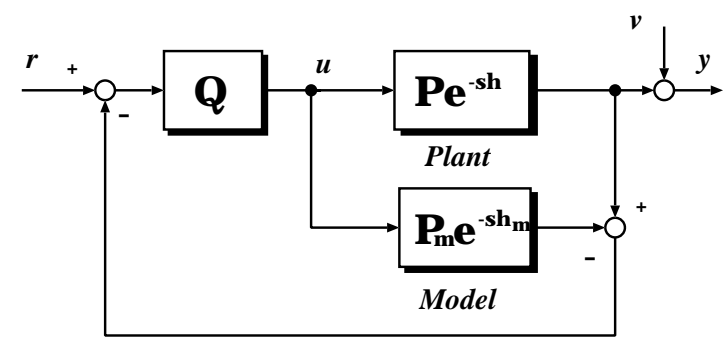

Figure 1: IMC Structure

IMC controller can be derived in the sense of $\mathcal{H}_{2}$ norm. The cost function of the IMC controller is expressed [11];

$$
\begin{aligned}
K_{I M C} & =\arg \min _{K} J_{I M C}(P, F), \\
J_{I M C}(P, F) & :=\left\|\frac{P e^{-s h} K}{1+P e^{-s h} K}-F e^{-s h}\right\|_{2},
\end{aligned}
$$

where, $P e^{-s h}$ denotes the plant and $K$ denotes ordinary unity feedback compensator. $K_{I M C}$ is represented as the following,

$$
\begin{aligned}
K_{I M C} & :=\arg \min _{K} J_{I M C}(P, F) \\
& =\frac{Q}{1-P e^{-s h} Q}
\end{aligned}
$$

Where, $Q$ is called IMC controller and $F(s)$ is called IMC filter, respectively. $F(0)=1$ is required to have no stationary error for step reference. Note that the ordinary unity feedback compensator has a time delay element. The IMC controller is easily derived by the plant and the IMC filter.

$$
\begin{aligned}
Q(s) & :=P_{-}^{-1}(s) F(s), \\
P(s) & :=P_{+}(s) P_{-}(s)
\end{aligned}
$$

$$
\begin{aligned}
& P_{+}(s): e^{-s h} \text { and non-minimum phase part of } P(s) \\
& P_{-}(s): \text { minimum phase part of } P(s)
\end{aligned}
$$

The IMC structure is shown in Fig.1. The closed-loop includes the plant model $P_{m} e^{-s h_{m}}$ and the difference between the plant and the model is used to control. This structure can be easily modified to the unity feedback structure with the compemsator (2) and Smith predictor controller [1]. (see Fig. 2.)

The controller $K$ is synthesized by the model and the plant parameters are unknown in generally. The cost function is rewritten as inequality;

$$
\begin{gathered}
J_{I M C}(P, F)=\left\|\frac{P e^{-s h} K}{1+P e^{-s h} K}-F e^{-s h_{m}}\right\|_{2} \\
=\| \frac{P e^{-s h} K}{1+P e^{-s h} K}-\frac{P_{m} e^{-s h_{m}} K}{1+P_{m} e^{-s h_{m}} K} \\
+\frac{P_{m} e^{-s h_{m}} K}{1+P_{m} e^{-s h_{m}} K}-F e^{-s h_{m}} \|_{2} \\
\leq\left\|\frac{P e^{-s h} K}{1+P e^{-s h} K}-\frac{P_{m} e^{-s h_{m}} K}{1+P_{m} e^{-s h_{m}} K}\right\|_{2} \\
\quad+\left\|\frac{P_{m} e^{-s h_{m}} K}{1+P_{m} e^{-s h_{m} K}}-F e^{-s h_{m}}\right\|_{2},
\end{gathered}
$$

where, $P_{m}$ and $e^{-s h_{m}}$ denote the lumped part of the model and the time delay of the model. The second term is rewritten as $J_{I M C}\left(P_{m}, F\right)$ and becomes small by IMC controller. On the other hand, the first term is minimized by the closed-loop identification of the prediction error method. Then a new model is derived.

$$
\begin{gathered}
P_{m}^{\text {new }}=\arg \min _{P} J_{I M C}^{i d}\left(P_{m}, F\right) \\
J_{I M C}^{i d}\left(P_{m}, F\right):=\left\|\frac{P e^{-s h} K}{1+P e^{-s h} K}-\frac{P_{m} e^{-s h_{m}} K}{1+P_{m} e^{-s h_{m}} K}\right\|_{2}
\end{gathered}
$$

By using iterative design method, both first and second terms of (4) are expected to derive a good closed-loop performance.

\subsection{Time delay identification}

When the time delay is unknown, one of the simplest method to decide the time delay is a coefficients comparison method for high order identification model [13]. However, it is not appreciated to minimize the prediction error.

At first we fix the order of the lumped part, we estimate parameters for each time delay and derive each loss function with the cross validation [13]. Then we set the time delay as the value of the smallest loss function and lumped parameters at this case. The loss function $V_{d}$ denotes the cost function of the prediction error itself depend on each time delay $d$.

$$
\begin{aligned}
V_{d} & =\frac{1}{N} \sum_{i=1}^{N} \varepsilon_{d}^{2}\left(k, \theta_{d}\right) \\
\varepsilon_{d}\left(k, \theta_{d}\right) & =A_{d}(q) y(k)-q^{-d} B_{d}(q) u(k),
\end{aligned}
$$

where, the estimate parameter vector $\theta_{d}$ (i.e. $A_{d}$ and $B_{d}$ ) of the lumped part for each time delay is estimated by the estimate data and the loss function $V_{d}$ is calculated from the verification data and each time delay.

Note that the above time delay identification will include not only pure time delay but also unmodeled high order slow dynamics. 


\subsection{Design Algorithm [5]}

We assume that the input-output relation of the plant is described as the following SISO discrete linear input time delay system;

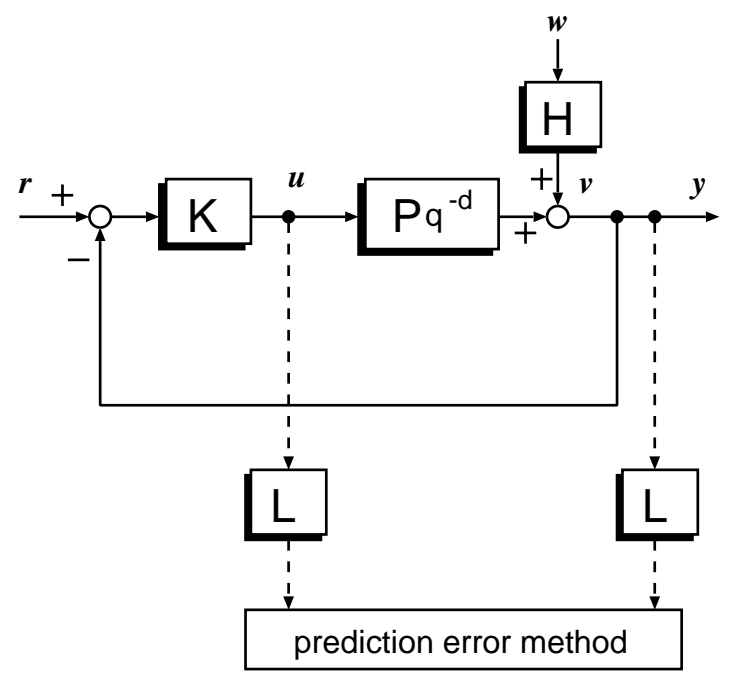

Figure 2: Closed-loop Identification of IMC

$$
y(k)=P(q) q^{-d} u(k)+H(q) w(k),
$$

where, $P(q), d$ and $H(q)$ denote plant, time delay of the plant and noise system, $u(k)$ and $y(k)$ denote input and output, which can be mesured. $v$ denotes observation noise, which is generated by white noise $w(k)$ fed through $H . r(k)$ is a step reference signal in control stage and identification signal (Pseudo Random Binary Signal) in the closed-loop identification stage.

The model is described as the following;

$$
y_{m}(k)=P_{m}(q, \theta) q^{-d_{m}} u(k)+H_{m}(q, \theta) w(k),
$$

where, subscribe $m$ denotes estimate model and $\theta$ denotes unknown parameter vector. The plant and the model are applied the IMC feedback compensator $K$ as

$$
\begin{aligned}
& u(k)=K(q)(r(k)-y(k)) \\
& y(k)=\left(1+P(q) q^{-d} K(q)\right)^{-1} \\
& \cdot\left\{P(q) q^{-d} K(q) r(k)+v(k)\right\} \\
& y_{m}(k)=\left(1+P_{m}(q, \theta) q^{-d_{m}} K(q)\right)^{-1} \\
& \cdot\left\{P_{m}(q, \theta) q^{-d_{m}} K(q) r(k)+v_{m}(k)\right\}
\end{aligned}
$$

Then one step prediction value of (13) is manipulated;

$$
\begin{aligned}
y_{m}(k \mid \theta)= & {\left[1-H_{m}^{-1}(q, \theta)\left(1+P_{m}(q, \theta) q^{-d_{m}} K(q)\right)\right] y(k) } \\
+ & H_{m}^{-1}(q, \theta)\left(1+P_{m}(q, \theta) q^{-d_{m}} K(q)\right) \\
& \cdot\left(1+P_{m}(q, \theta) q^{-d_{m}} K(q)\right)^{-1} \\
& \cdot P_{m}(q, \theta) q^{-d_{m}} K(q) r(k)
\end{aligned}
$$

One step prediction error is described;

$$
\begin{aligned}
\varepsilon(k, \theta)= & y(k)-y_{m}(k \mid \theta) \\
= & \frac{1+P_{m}(q, \theta) q^{-d_{m}} K(q)}{H_{m}(q, \theta)}\{ \\
& {\left[\frac{P(q) q^{-d} K(q)}{1+P(q) q^{-d} K(q)}\right.} \\
& \left.-\frac{P_{m}(q, \theta) q^{-d_{m}} K(q)}{1+P_{m}(q, \theta) q^{-d_{m}} K(q)}\right] r(k) \\
& \left.+\frac{H(q)}{1+P(q) q^{-d} K(q)} w(k)\right\}
\end{aligned}
$$

$r(k)$ and $w(k)$ are assumed to be mutually uncorrelated, the prediction error problem with a frequency weighting function is;

$$
\begin{aligned}
& \lim _{N \rightarrow \infty} \frac{1}{N} \sum_{k=1}^{N}\{L(q) \varepsilon(k, \theta)\}^{2} \\
= & \frac{1}{2 \pi} \int_{-\pi}^{\pi}\left\{\mid \frac{P(j \omega) e^{-j \omega h} K(j \omega)}{1+P(j \omega) e^{-j \omega h} K(j \omega)}\right. \\
& -\left.\frac{P_{m}(j \omega, \theta) e^{-j \omega h_{m}} K(j \omega)}{1+P_{m}(j \omega, \theta) e^{-j \omega h_{m}} K(j \omega)}\right|^{2} \Phi_{r}(\omega) \\
+ & \left.\left|\frac{H(j \omega)}{1+P(j \omega) e^{-j \omega h} K(j \omega)}\right|^{2} \Phi_{w}(\omega)\right\} \\
& \cdot \frac{L(j \omega)\left(1+P_{m}(j \omega, \theta) e^{-j \omega h_{m}} K(j \omega)\right)}{H_{m}(j \omega, \theta)} d \omega
\end{aligned}
$$

where, $\Phi_{r}$ and $\Phi_{w}$ denote power spectrum of the identification signal $r$ and the noise $w$. To derive a $P_{m}(q, \theta)$ to minimize the above criterion. When $L(q)$ is chosen as the following;

$$
L(q)=\frac{H_{m}(q, \theta)}{1+P_{m}(q, \theta) q^{-d_{m}} K(q)}
$$

And it is satisfied $\Phi_{r}(\omega)=1 \gg \Phi_{w}(\omega)$, since $r$ is the identification signal (PRBS) in this identification stage,

$$
\begin{aligned}
& \lim _{N \rightarrow \infty} \frac{1}{N} \sum_{k=1}^{N}\{L(q) \varepsilon(k, \theta)\}^{2} \\
= & \| \frac{P(j \omega) e^{-j \omega h} K(j \omega)}{1+P(j \omega) e^{-j \omega h} K(j \omega)} \\
& -\frac{P_{m}(j \omega, \theta) e^{-j \omega h_{m}} K(j \omega)}{1+P_{m}(j \omega, \theta) e^{-j \omega h_{m}} K(j \omega)} \|_{2}^{2}
\end{aligned}
$$

It is the same criterion as the identification cost function (6). In the closed-loop identification, $L(q)$ is used as the pre-filter. $L(q)$ depends on the previous controller designed and not the current controller. This is an inherent feature of iterative design method.

We summarize the joint design method of IMC controller and closed-loop identification for input time delay systems. 
step 1 (Pre-identification) estimate nearly model $P_{m}(s) e^{-s h_{m}}$ of the plant $P(s) e^{-s h}$ and time delay with open-loop identification such as step response.

$$
\begin{aligned}
P_{m 1} & =\arg \min _{P_{m}} J_{\text {open }}^{i d}\left(P_{m}\right) \\
J_{\text {open }}^{i d} & :=\left\|P(s) e^{-s h}-P_{m}(s) e^{-s h_{m}}\right\|_{2}
\end{aligned}
$$

step 2 (IMC compensator design) set the time constant $\lambda$ of the IMC filter (1) and the integer $n$ and derive the IMC compensator as in the section 2.1.

step 3 (Experiment of control) The step reference input is input from $r$ and derive the closed-loop response. If the closed-loop has desired performance, then the step is ended here. If not, it goes on the following step.

step 4 (Experiment of closed-loop identification) The identification signal (PRBS) is input from $r$. Input data for the closed-loop identification is derived the output of controller $K$ and output signal is received from output $y$. (show Fig.2)

step 5 (Parameter estimation) estimate the model $P_{m}(s) e^{-s h_{m}}$ of the plant. Input-output data of the closed-loop (show Fig.2) is filtered by the pre-filter $L(q)$ (17) and the model $P_{m(i+1)}$ is derived by the prediction error method (least square method) and time delay identification method as in the section 2.2. Then go back to step 2 .

This joint design is continued until closed-loop has desired performance.

\section{Experimental Results}

Experiment equipment is a cooling device with a frigistor module. The control purpose is to cool water at target temperature as like step response by frigistor. The target temperature is $-3^{\circ}$ from the room temperature. The system is a single input and single output system, and the experimental model is shown in Fig. 3. It has a small time delay, however, in order to make the effectiveness clear, artificial time delay 50 [s] is inserted by software. The inserted time delay is not so long compared with the system time constant.

\subsection{PI Control}

At the beginning, we show the ordinary PID control result in Fig. 4. The response is a typical response of the temperature control. It has an overshoot and small oscillation. In the temperature control, the overshoot is disliked, therefore, it is required the other controller which is considered time delay.

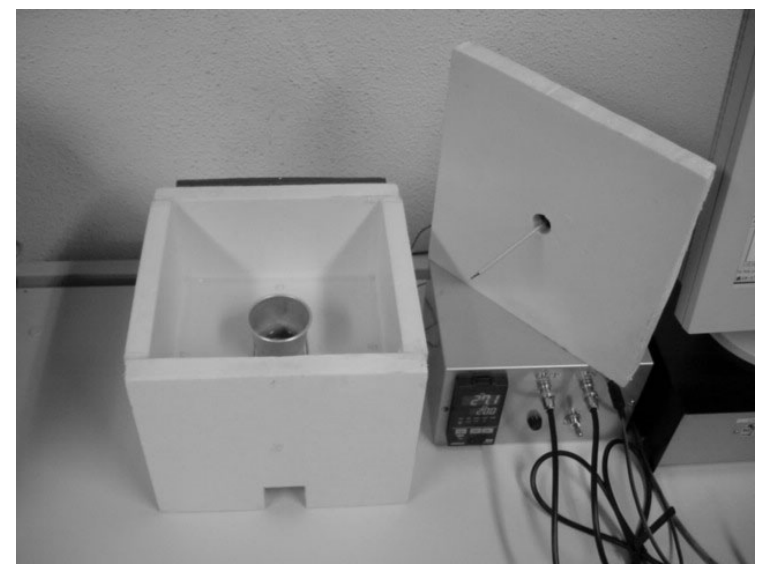

Figure 3: Temperature control experimental model

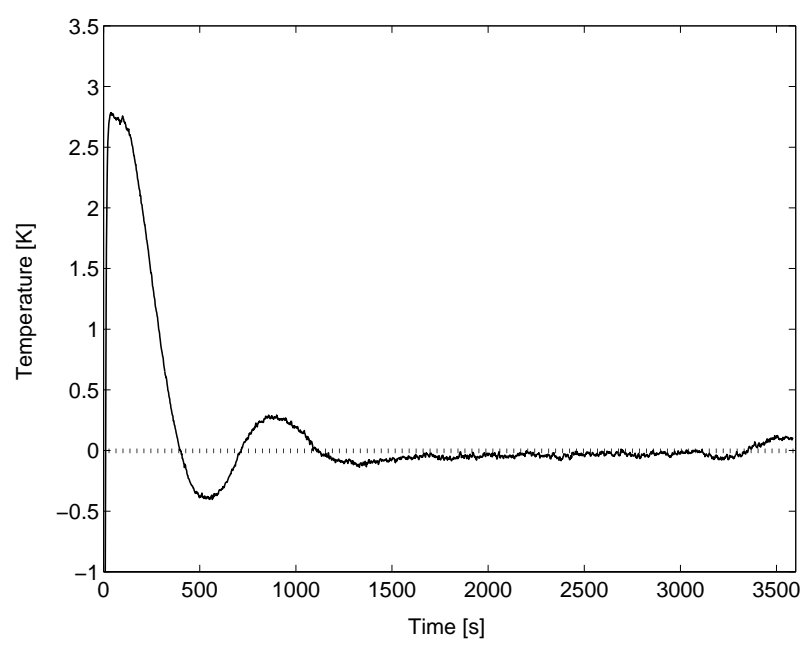

Figure 4: Experimental result of PID

\subsection{Iterative Control}

In step 1, the pre-model of the plant is described as the following transfer function with an articicial input time delay.

$$
P(s)=\frac{-3.19}{968 s+1} e^{-s h}, \quad h=50
$$

Fig. 5 shows a step response without artificial time delay. Ordinary case, it is modeled simple 1 st order system. The step inpu signal is set about the target temperature, i.g. $-3^{\circ}$ from room temperature. In Fig. 5, $0^{\circ}$ denotes the room temperature.

In step 2, the time constant of IMC filter $\lambda$ set $\lambda=800$ and $n=1$. The time constant of the pre-model (21) is an indicate to set the time constant of IMC filter. When the time constant is set smaller, then the bandwidth becomes larger, however, the closed-loop property becomes sensitive. On the contrary, the time constant is set larger than the open-loop time constant, the closed-loop property is robust and has a loose response. Then we derive and set the IMC compensator with $\lambda$ by using (3).

Fig.6 is an experimental result with pre-model (step 3, iteration=0). In this figure, the target temperature is normalized 


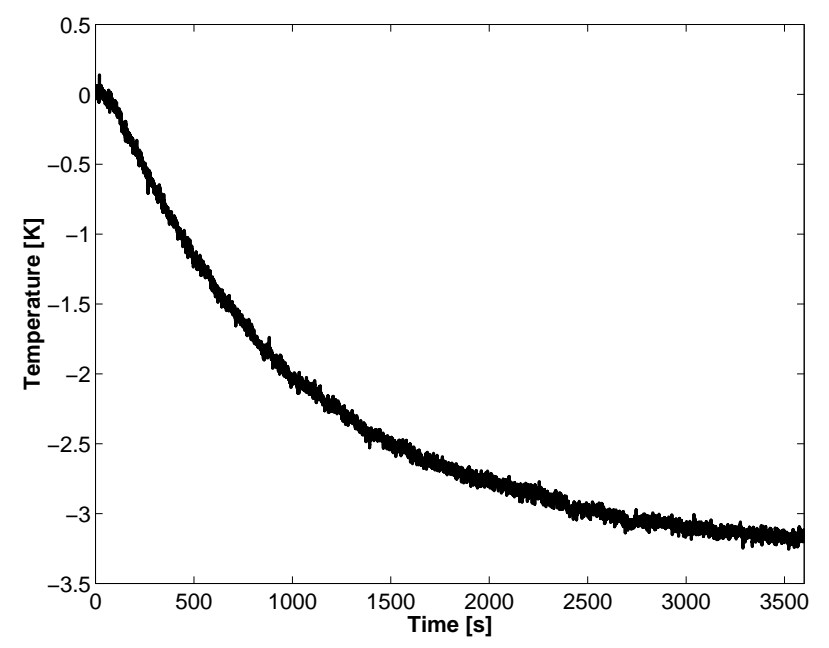

Figure 5: Step response without inserted time delay

at 0 . The response constructed by pre-model has overshoot, long time oscillation and the rising time is too long. Although the time delay is smaller than the open-loop time constant and the step response seems very simple, as shown Fig.6, the closed-loop performance is not good. In IMC structure, the closed-loop property is affected by the model immediately, the re-model is better way rather than tuning the free parameter. Therefore we go on the next step, closed-loop identification.

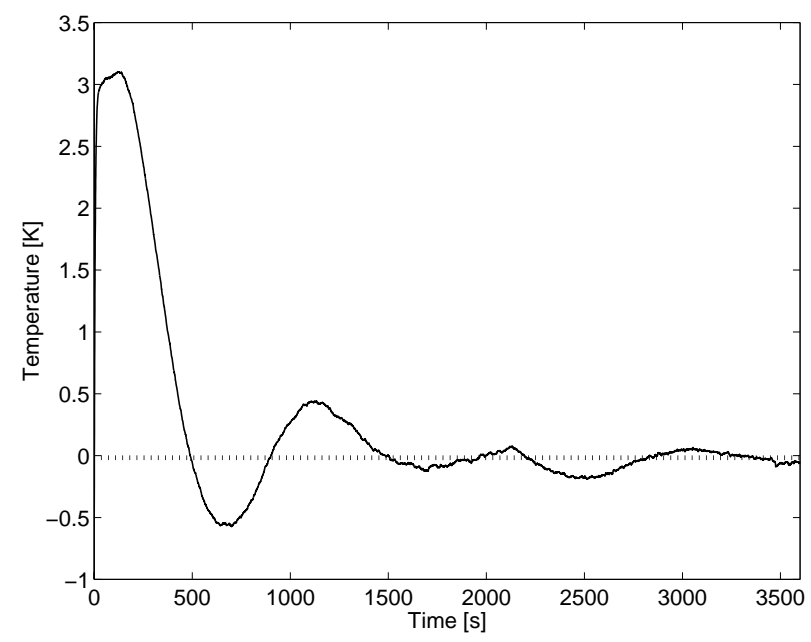

Figure 6: Experimental result of IMC (iteration=0)

Fig.7 and Fig.8 are closed-loop identification results (step 4 and $\mathbf{5}$, iteration=1). Here, conditions for closed-loop identification are as follows;

Model: 2nd order DARX model

Input: PRBS (shift register: $\mathrm{n}=10$, iterate number $l=2$ )

Data number: $N=2^{n} l-1$ )

Id. sampling time: $1[\mathrm{~s}]$
Fig.7 shows loss function $V_{d}$ for each time delay between 20 [s] and 300 [s] with cross validation. In Fig.7, the loss function becomes rapidly small on the point considered to be time delay. The minimum value is not garanteed the global minimum in the theoretical sense, however, considering the plant behavior, the value is valid minimum point.

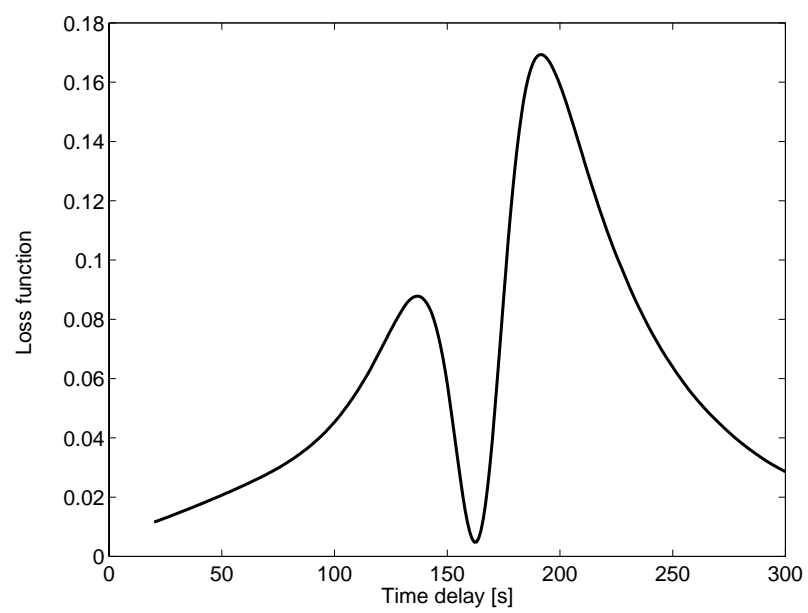

Figure 7: Loss function of time delay (iteration=1)

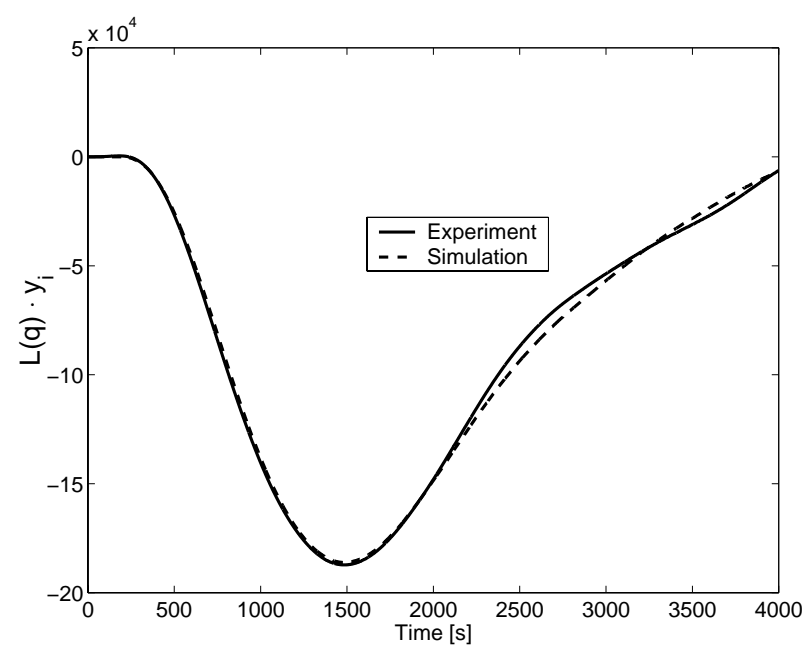

Figure 8: Certainty of model (iteration=1)

Fig.8 shows certainty of estimated model by comparing simulation result of the model with experimental result. A vertical axis is filtered identification output $y_{i}$ by the pre-filter $L(q)$ (17). From Fig.8, it can be stated that the estimated model coincides approximately with the experimental result.

The estimated model of the plant is described as the following transfer function with an input time delay.

$$
\begin{aligned}
P_{1}(s)= & \frac{0.002522 s^{2}-0.005035 s-1.629 \times 10^{-5}}{s^{2}+0.003495 s+3.065 \times 10^{-6}} e^{-s h_{1}}, \\
& h_{1}=162,
\end{aligned}
$$


where time delay $h_{1}=162$ is different from inserted time delay $h=50$. The difference is too big even if it is assumed that delay of rising is contained in time delay $h_{1}$. It can be stated that $h_{1}$ is not exact value, however, the performance in the sence of $\mathrm{H}_{2}$ norm is better than open-loop identification by step response.

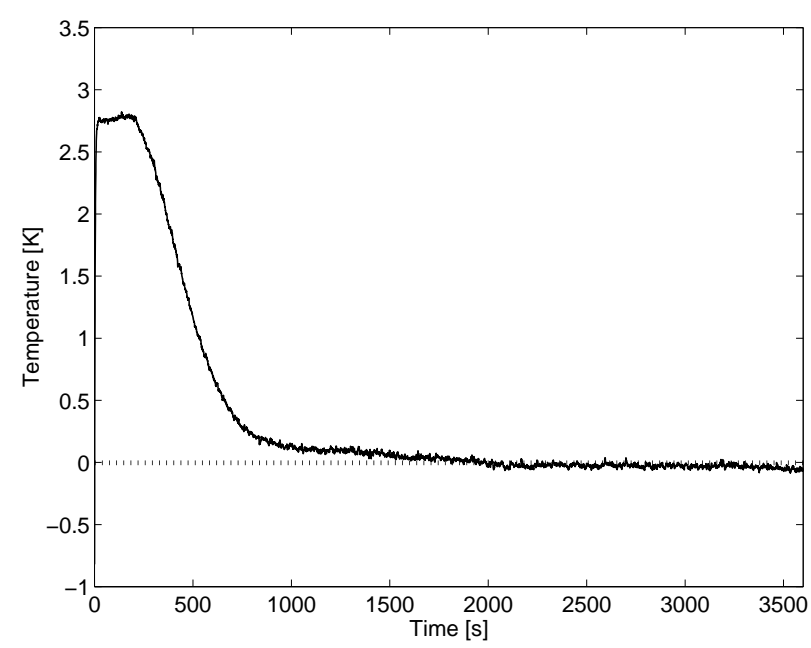

Figure 9: Experimental result of IMC (iteration=1)

Fig.9 is experimental result with the model (iteration=1), here $\lambda$ is equal to the value of the first iteration. This figure shows that closed-loop performance is quite improved without overshoot and rising time is short compared with Fig.6. It can be stated that high closed-loop performance is obtained even if exact time delay is not estimated. In other words, the obtained model (22) is a suitable synthesis model for IMC structure.

After the experiment, identification and control experiment are repeated several times. However, any results were the same response. Therefore, those results are omitted.

\section{Conclusion}

We demonstrate a joint design method of closed-loop identification and IMC for temperature control system with input time delay. Experimental results substantiated validity of the method. And, the closed-loop has good performance with easily parameter setting compared with ordinary PID control.

In our experience, decimation and interpolation should not be done at the closed-loop identification stage. Because these processing affect bad precision of the loss function. It is not good for identification of time delay.

Considering the simplicity of the model, experimental results has good performance. The iterative algorithm is just complex for the engineer on the spot, we will consider an auto tuning method by using this algorithm.

\section{References}

[1] N.Abe and K.Yamanaka: Smith Predictor Control and Internal Model Control - A Tutorial -, SICE Annual Conference, Fukui (2003) to appear

[2] H.Ichihara and N.Abe: Iterative Identification and LQG Control Design for Time Delay Systems, Transactions of the Institute of Systems, Control and Information Engineers, Vol.15, No.4, pp.159-166 (2002) (In Japanese).

[3] P.Albertos and A.Sala (Eds.): Iterative Identification and Control, Springer (2002)

[4] H.Ichihara, Y.Matsumoto, N.Abe and H.Kanoh: Application of an Iterative Identification and Control Design to a Vibration System, JSME International Journal, Series C, Vol.43, No.1, pp.18-24 (2000).

[5] N.Abe and H.Ichihara: Closed-loop Identification and IMC Design for Systems with an Input Time Delay, ECC99, F0362 (1999)

[6] P.M.J.Van Den Hof and R.J.P.Schrama: Identification and Control - Closed-loop Issues, Automatica, Vol.31, No.12, pp.1751-1770 (1995).

[7] W.S.Lee, B.D.O.Anderson, I.M.Y.Mareels and R.L.Kosut: On Some Key Issues in the Windsurfer Approach to Adaptive Robust Control, Automatica, Vol.31, No.11, pp.16191636 (1995).

[8] H.Hjalmarsson, S.Gunnarsson, and M.Gevers: Optimality and sub-optimality of iterative identification and control schemes, Proc. ACC, pp.2559-2563 (1995)

[9] R.G.Hakvoort, R.J.P.Schrama and P.M.J.Van Den Hof: Approximate Identification with Closed-loop Performance Criterion and Application to LQG Feedback Design, Automatica, Vol.30, No.4, pp.679-690 (1994).

[10] H.Hjalmarsson, M.Gervers, F.de Bruyne and J.Leblond: Identification for Control: closing the loop gives more accurate controllers, Proc. 33rd CDC, pp.4150-4155 (1994)

[11] M.Morari: Robust Process Control, Prentice Hall (1989).

[12] F.Hansen and G.Frankin: On a Fractional Representation Approach to Closed-Loop Experiment Design, Proc. of ACC, pp.1319-1320 (1988).

[13] L.Ljung: System Identification: Theory for the User, Prentice Hall (1987) 